\title{
Lung Abscess Caused by Actinomyces odontolyticus
}

\author{
Yasuo TAKIGUCHI, Takashi TERANO and Akira HIRAI
}

\begin{abstract}
A 64-year-old woman with periodontal disease was admitted because of fever, right chest pain, and bloody sputum. Her chest radiograph revealed a nodular shadow in the right middle lung fields. From the results of a needle aspiration biopsy, she was diagnosed with a lung abscess due to Actinomyces odontolyticus (A. odontolyticus) and Streptococcus spp. The patient improved quickly with antibiotics. To our knowledge, only nine cases of pulmonary infection due to $A$. odontolyticus have been reported, and we report the first case in Japan. Although respiratory disease caused by $A$. odontolyticus is rare, the association of this organism should be considered even in healthy individuals.
\end{abstract}

(Internal Medicine 42: 723-725, 2003)

Key words: lung abscess, Actinomyces odontolyticus, periodontal disease, ampicillin sodium/sulbactam

\section{Introduction}

Actinomycosis is an uncommon diagnosis, and furthermore, pulmonary actinomycosis is rare. Actinomyces israelii is the primary pathogen that causes actinomycosis and pulmonary infection due to Actinomyces odontolyticus (A. odontolyticus) is very rare (1-3). This organism is found in normal buccal flora and is an important pathogen of dental caries. Only nine cases of pulmonary infection due to $A$. odontolyticus have been reported (4-11), and none were from Japan. We report a case of lung abscess caused by $A$. odontolyticus and Streptococcus spp.

\section{Case Report}

A 64-year-old woman was admitted to our hospital because of an abnormal pulmonary mass shadow. She had been receiving treatment for periodontal disease at a dental clinic since January 20, 2002. Fever, right chest pain, and bloody sputum developed on February 11. Her symptoms persisted, and she had a medical visit on February 15 . Chest radiographs revealed a nodular shadow in the right middle lung fields. On the same day, she was transferred to our hospital. Her medical history included appendictitis at age 33 years. She denied smoking cigarettes, using steroids, and abusing alcohol. The patient also did not have diabetes or other underlying diseases.

Findings on physical examination included temperature $38.0^{\circ} \mathrm{C}$, pulse rate 68 beats per minute, and respirations rate 20 breath per minute. Her blood pressure was 126/68 mmHg. The patient was $155 \mathrm{~cm}$ tall and weighed $58 \mathrm{~kg}$. The oxygen saturation was 97 percent while the patient was breathing ambient air. On physical examination, the head, neck, and skin were normal. There was no lymphadenopathy. Respiratory and heart sounds were normal. Examination of the abdomen and neurologic system were unremarkable.

Laboratory test data revealed a white blood cell count of $10,700 / \mathrm{mm}^{3}$ with $74 \%$ neutrophils, hemoglobin of $11.4 \mathrm{~g} / \mathrm{dl}$, platelet count of $25.1 \times 10^{4} / \mathrm{mm}^{3}$, erythrocyte sedimentation rate of $92 \mathrm{~mm}$ per hour, and C-reactive protein of 10.6 $\mathrm{mg} / \mathrm{dl}$. Results of liver and renal function studies were normal. Chest radiographs (Fig. 1) showed a pulmonary nodule in the right upper lobe. Chest computed tomographic (CT) examination (Fig. 2) revealed a round mass in the right $\mathrm{S}^{3}$. Cavitation, pleural effusion, or lymph adenopathy were not detected. The chest wall and ribs appeared normal.

Percutaneous needle aspiration of the mass obtained dark pus. Microscopic examination of the specimen showed gram-positive coccus with numerous neutrophils, and ZiehlNeelsen staining was negative. Sulfur granules were not found. Bacterial colonies grew anaerobic gram-positive rods and aerobic gram-positive coccus. The former were $A$. odontolyticus identified by the Rap ID ANA II system (Version 5.93 Remel Co., Atlanta, GA, USA), and the latter were Streptococcus spp. Therefore, a lung abscess due to A. odontolyticus and Streptococcus spp. was diagnosed.

The patient received $3 \mathrm{~g}$ of ampicillin sodium/sulbactam sodium intravenously twice daily. On the fourth hospital day,

From the Department of Internal Medicine, Chiba Municipal Hospital, Chiba Received for publication December 10, 2002; Accepted for publication April 16, 2003

Reprint requests should be addressed to Dr. Yasuo Takiguchi, the Department of Internal Medicine, Chiba Municipal Aoba Hospital, 1273-2 Aoba, Chuoku, Chiba 260-0852 


\section{TAKIGUCHI et al}

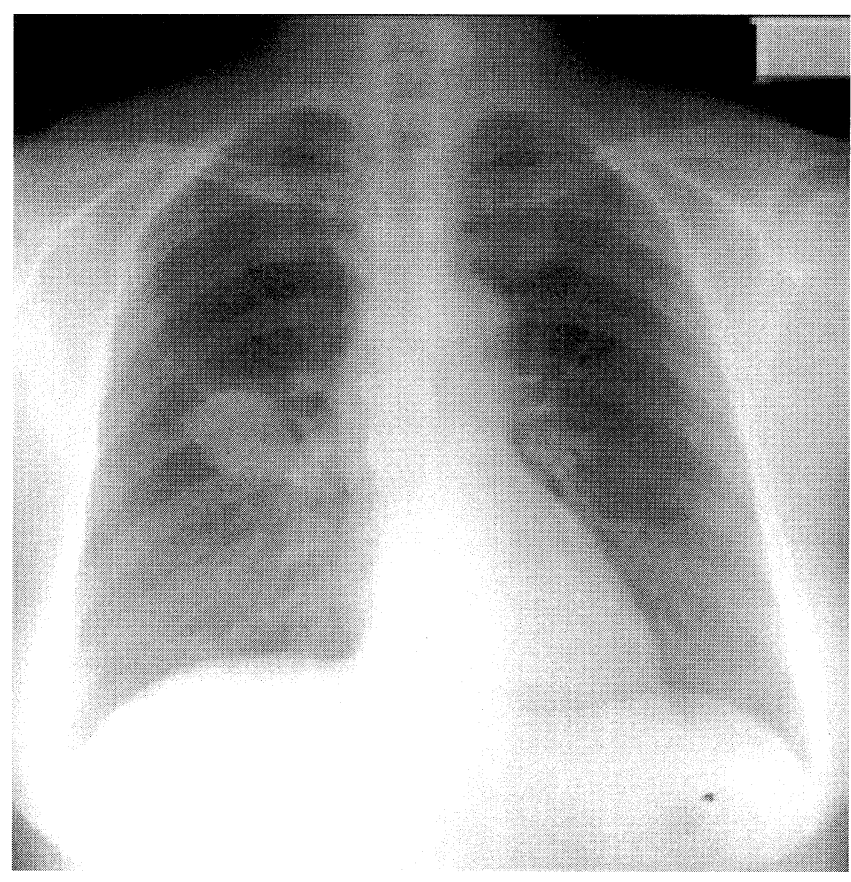

Figure 1. Chest radiograph showing nodular shadow in the right middle lung fields.

her temperature dropped and the white blood cell count and C-reactive protein decreased to $8,000 / \mathrm{mm}^{3}$ and $7.9 \mathrm{mg} / \mathrm{dl}$, respectively. White blood cell count and C-reactive protein were $6,300 / \mathrm{mm}^{3}$ and $4.5 \mathrm{mg} / \mathrm{dl}$, respectively on the sixth hospital day, and the patient's condition has improved. Her chest radiographs showed the nodular shadow had decreased. Antibiotic treatment was changed to oral slutamicillin tosilate $1,125 \mathrm{mg}$ daily and she was discharged on February 20. At an outpatient visit on February 27, the white blood cell count and C-reactive protein level had returned to normal. Chest CT images revealed a small residual nodular shadow. She continued treatment with her primary care physician.

\section{Discussion}

Actinomyces organisms are gram-positive or facultative anaerobes and are not highly virulent. They normally are found in the human oropharynx, particularly in persons with poor oral hygiene. Pulmonary actinomycosis is thought to result from aspiration of these organisms into the lungs. Risk factors include predisposition to aspiration, alcohol abuse, periodontal diseases, and carious teeth. In the present case, two causative pathogens were detected and we suspect that lung aspiration was the route of infection.

Primary actinomycosis confirmed by a microscopic examination and the presence of characteristic sulfur granules seen with Actinomyces israelii. However, these granules are absent in A. odontolyticus infections. Additionally, positive sputum culture cannot establish a diagnosis of pulmonary

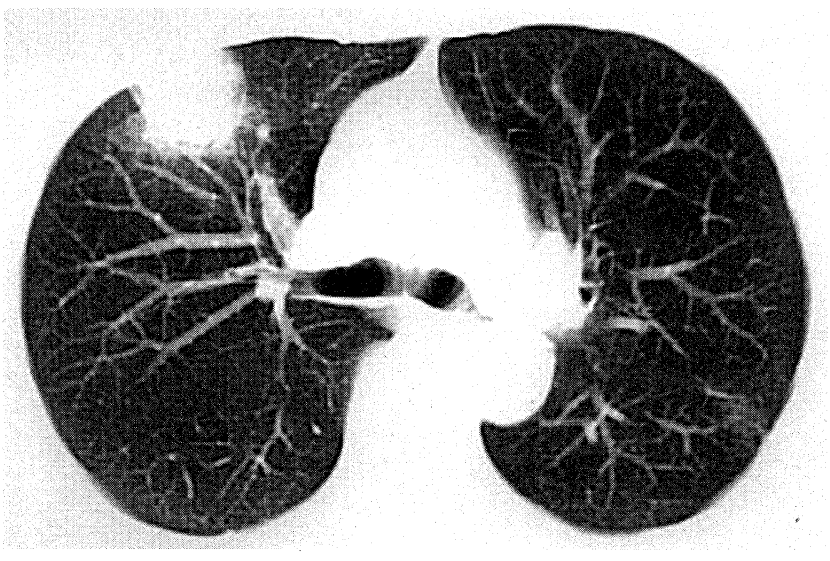

Figure 2. Chest computed tomography showing a nodular shadow in the right $S^{3}$.

actinomycosis because $A$. odontolyticus is present in normal oropharyngeal flora $(1,2,12)$. In this case, the diagnosis was confirmed by percutaneous aspiration biopsy before antibiotics were administered. We conclude that $A$. odontolyticus was one of the causative pathogens, and the patient improved with the appropriate antibiotic therapy.

A. odontolyticus, a commensal organism found in the mouth, was first isolated from dental caries in 1958 (13). A. odontolyticus is an important pathogen of dental caries. The first report of this organism causing clinical infection was in a case of large subcutaneous abscess (14). Since then, the organism has been isolated from patients with various infections; however, thoracopulmonary infection due to $A$. odontolyticus is rare and only nine cases have been reported in the English (3-10) and non-English (15-17) language literature.

Although guidelines for appropriate antibiotic treatment of thoracic actinomycosis remain undefined due to the disease rarity and lack of controlled trials, the recommended therapy for actinomyces infection is 4 to 6 weeks of intravenous penicillin followed by a 6 to 12 month oral regimen (3). We changed to oral penicillin after 6 days of intravenous treatment and the clinical course was successful. This antimicrobial regimen, which has not been established, might be a promising alternative to the long-term treatment with intravenous and oral penicillin.

Although respiratory infection caused by A. odontolyticus is rare, evaluation for this organism, which causes periodontal disease, should be considered even in healthy individuals. Maintenance of orodental hygiene is an important preventive measure against lung abscess resulting from aspiration of oropharyngeal flora into the lower respiratory tract.

We gratefully acknowledge the assistance of Kori Y. and Suruga Y. in our hospital. 


\section{References}

1) Heffner JE. Pleuropulmonary manifestations of actinomycosis and nocardiosis. Semin Respir Infect 3: 352-361, 1988.

2) Hsieh MJ, Liu HP, Chang JP, Chang $\mathrm{CH}$. Thoracic actinomycosis. Chest 104: 366-370, 1993.

3) Smego RA Jr, Foglia G. Actinomycosis. Clin Infect Dis 26: 12551263, 1998.

4) Baron EJ, Angevine JM, Sundstrom W. Actinomycotic pulmonary abscess in an immunosuppressed patient. Am J Clin Pathol 72: 637-639, 1979.

5) Bellingan GJ. Disseminated actinomycosis. BMJ 301: 1323-1324, 1990.

6) Hooi LN, Na BS, Sin KS. A case of empyema thoracis caused by actinomycosis. Med J Malaysia 47: 311-315, 1992.

7) Dontfraid F, Ramphal R. Bilateral pulmonary infiltrates in association with disseminated actinomycosis. Clin Infect Dis 19: 143-145, 1994.

8) Bassiri AG, Girgis RE, Theodore J. Actinomyces odontolyticus thoracopulmonary infections. Two cases in lung and heart-lung transplant recipients and a review of the literature. Chest 109: 1109-1111, 1996.

9) Perez-Castrillon JL, Gonzalez-Castaneda C, del Campo-Matias F, Bellido-Casado J, Diaz G. Empyema necessitates due to Actinomyces odontolyticus. Chest 111: 1144, 1997.

10) Iancu D, Chua A, Schoch PE, Cunha BA. Actinomyces odontolyticus pulmonary infection. Am J Med 107: 293-294, 1999.

11) Litwin KA, Jadbabaie F, Villanueva M. Case of pleuropericardial disease caused by Actinomyces odontolyticus that resulted in cardiac tamponade. Clin Infect Dis 29: 219-220, 1999.

12) Holmberg K. Diagnostic methods for human actinomycosis. Microbiol Sci 4: 72-78, 1987.

13) Batty I. Actinomyces odontolyticus, a new species of actinomycete regularly isolated from deep carious dentine. J Pathol Bacteriol 75: 455-459, 1958.

14) Morris JF, Kilbourn P. Systemic actinomycosis caused by Actinomyces odontolyticus. Ann Intern Med 81: 700, 1974.

15) Raoult D, Kohler JL, Gallais H, Estrangin E, Peloux Y, Casanova P. Fusobacterium necrophorum associated with Actinomyces odontolyticus septicemia. Pathol Biol (Paris) 30: 576-580, 1982 (in French, Abstract in English).

16) Verrot D, Disdier P, Harle JR, et al. Pulmonary actinomycosis: is Actinomyces odontolyticus responsible? Rev Med Intern 14: 179-181, 1993 (in French, Abstract in English).

17) Ibanez-Nolla J, Carratala J, Cucurull J, et al. Thoracic actinomycosis. Enferm Infecc Microbiol Clin 11: 433-436, 1993 (in Spanish, Abstract in English). 